\title{
An Interval Probability-based Inexact Two-stage Stochastic Model for Regional Electricity Supply and GHG Mitigation Management under Uncertainty ${ }^{*}$
}

\author{
Yulei Xie, Guohe Huang, Wei Li, Ye Tang \\ North China Electric Power University, Key Laboratory of Regional Energy System Optimization, \\ Ministry of Education, Beijing, China \\ Email: xieyulei850228@yahoo.com, guohe.huang3@gmail.com,weili819@yahoo.com.cn,tye0202@hotmail.com
}

Received October, 2012

\begin{abstract}
In this study, an interval probability-based inexact two-stage stochastic (IP-ITSP) model is developed for environmental pollutants control and greenhouse gas (GHG) emissions reduction management in regional energy system under uncertainties. In the IP-ITSP model, methods of interval probability, interval-parameter programming (IPP) and two-stage stochastic programming (TSP) are introduced into an integer programming framework; the developed model can tackle uncertainties described in terms of interval values and interval probability distributions. The developed model is applied to a case of planning GHG -emission mitigation in a regional electricity system, demonstrating that IP-ITSP is applicable to reflecting complexities of multi-uncertainty, and capable of addressing the problem of GHG-emission reduction. 4 scenarios corresponding to different GHG -emission mitigation levels are examined; the results indicates that the model could help decision makers identify desired GHG mitigation policies under various economic costs and environmental requirements.
\end{abstract}

Keywords: Interval Probability; Inexact Two-stage Stochastic Programming; Electricity Generation; GHG-Mitigation; Energy System

\section{Introduction}

For many decades, the constantly increment of regional electricity demand has forced managers to contemplate and propose ever more comprehensive, complex and ambitious plans for electric power systems. However, most $\mathrm{CO}_{2}$ emissions are emitted mainly from electricity generation processes of burning fossil fuels such as coal, oil and natural gas [1]. A number of impact factors, such as population growth, rapid urbanization and industrialization, and global economic development, would inevitably result in conflicts among economic objective, electricity demand/supply, and environmental requirement. Moreover, such planning efforts are complicated with a variety of uncertain parameters as well as their interactions [2]. It is thus deemed necessary to develop effective optimization methods for supporting regional electric power systems management with GHG-emission mitigation under such complexities.

Previously, a number of mathematical techniques have been introduced to deal with these uncertainties [3-6].

*Supported by Major Science and Technology Program for Water Pollution Control and Treatment (NO. 2009ZX07631-03).
Among these techniques, inexact two stage stochastic programming model (ITSP) integrated with interval parameter programming (IPP) and two stage stochastic programming (TSP) has received extensive attentions to express uncertain parameters with random and dynamic feature as probability distributions and discrete intervals over the past years [7]. Generally, all of the stochastic programming models require probabilistic specifications for uncertain parameters. However, in many practical situations, discrete probability distributions of future events may be predicted using existing statistics and expert judgment. For long run decision making problems, these probabilities are in most cases subjective and difficult to estimate. For example, in electric power management system, it is often associated with difficulties in acquiring the probability distribution of the random variables/ parameters due to the insufficient available data or the existence of multiple uncertainties. When only limited or imprecise information is available while the stochastic programming method is used, the detailed probabilistic distributions need to be generated based on unrealistic assumptions, resulting in potential errors with the modeling inputs and outputs. Interval probability 
distribution, which was based on the interval numbers and stochastic mathematics method, was effective for dealing with uncertainties on probability distribution [8]. Interval probability distribution relates to the case where over the interval of possible probabilities some values are more possible than others. Previously, a number of inexact optimization methods coupled with interval probability distribution were developed for dealing with uncertainties presented as intervals and/or random variables [8]. Nevertheless, no previous study was reported on interval probability distribution-based two stage stochastic programming for GHG-emission management in regional electric power systems.

Therefore, as an extension of the pervious study, the objective of this study is to develop an interval probability distribution- based two stage stochastic programming (IP-TSP) and applies it to the planning of regional electric power systems with GHG-emission mitigation. The IP-TSP will integrate optimization techniques of interval parameter programming, two stage stochastic programming, and interval probability distribution into a general framework to handle multiple uncertainties. In a hypothetical regional electric power system, the developed method will be used to analyze various policy scenarios that are associated with different levels of economic consequences when the promised electricity generation targets are violated. The results will help managers to not only make decisions of electricity generation schemes but also gain insight into the tradeoffs between system risk and economic objectives.

\section{Methodology}

\subsection{Interval Probability Distribution}

Traditional methods of the stochastic decision making are based on the assumption that we know the probabilities of different situations ,In some situations, it would be difficult generating the exact value of the probabilities, and more uncertainties would exit in the energy systems to affect the probability distribution of the stochastic parameters. The interval would be able to effectively reflect the uncertainties of the possible value of probability. In order to make decisions under such interval probabilities, Reference [8] developed a natural way of decision making under interval probabilities. For example, there exists exactly one averaging operation with interval probabilities, and this averaging operation has the form:

$$
\begin{aligned}
& C\left\{\left(\left[p_{1}^{-}, p_{1}^{+}\right], c_{1}\right),\left(\left[p_{2}^{-}, p_{2}^{+}\right], c_{2}\right), \ldots,\left(\left[p_{n}^{-}, p_{n}^{+}\right], c_{n}\right)\right\} \\
& =p_{1} \cdot c_{1}+p_{2} \cdot c_{2}+\ldots+p_{n} \cdot c_{n}
\end{aligned}
$$

where

$$
p_{j}=\frac{\sum_{j=1}^{n} p_{j}^{+}-1}{\sum_{j=1}^{n} p_{j}^{+}-\sum_{j=1}^{n} p_{j}^{-}} \cdot p_{j}^{-}+\frac{1-\sum_{j=1}^{n} p_{j}^{-}}{\sum_{j=1}^{n} p_{j}^{+}-\sum_{j=1}^{n} p_{j}^{-}} \cdot p_{j}^{+}
$$

Thus, the interval probabilities can be transferred into deterministic probabilities.

\subsection{Interval Probability Distribution- based Two-stage Stochastic Programming}

In the two stage stochastic program, the conditional probabilities, are supposed to have a predefined exact value. However, in some situations these probabilities are difficult to evaluate and only an estimation of their possible value may be available. Therefore, these probabilities may be considered as interval probability distributions. Thus, an interval probability distribution- based inexact two-stage stochastic programming (IP-TSP) can be formulated as follow:

$$
\max f^{ \pm}=\sum_{j=1}^{n_{1}} c_{j}^{ \pm} x_{j}^{ \pm}-\sum_{j=1}^{n_{1}} \sum_{k=1}^{K} p_{k} d_{j}^{ \pm} y_{j k}^{ \pm}
$$

subject to:

$$
\begin{gathered}
\sum_{j=1}^{n_{1}} a_{r j}^{ \pm} x_{j}^{ \pm} \leq b_{r}^{ \pm}, r=1,2, \ldots, m_{1} \\
\sum_{j=1}^{n_{1}} a_{i j}^{ \pm} x_{j}^{ \pm}+\sum_{j=1}^{n_{1}} e_{i j}^{ \pm} y_{j k}^{ \pm} \leq \hat{w}_{i k}^{ \pm}, i=1,2, \ldots, m_{2} ; \\
k=1,2, \ldots, K \\
\beta_{k}^{-} \leq p_{k} \leq \beta_{k}^{+}, k=1,2, \ldots, K \\
\sum_{k=1}^{K} \beta_{k}^{+}-\sum_{k=1}^{K} \beta_{k}^{-} \\
\sum_{k}^{K} \beta_{k}^{+}+\frac{1-\sum_{k=1}^{K} \beta_{k}^{-}}{\sum_{k=1}^{K} \beta_{k}^{+}-\sum_{k=1}^{K} \beta_{k}^{-}} \cdot \beta_{k}^{+} \\
\sum_{k=1}^{K} p_{k}=1 \\
x_{j}^{ \pm} \geq 0, j=1,2, \ldots, n_{1} \\
y_{j k}^{ \pm} \geq 0, j=1,2, \ldots, n_{1} ; k=1,2, \ldots, K
\end{gathered}
$$

where $p_{k}=\left[\beta_{k}^{-}, \beta_{k}^{+}\right]$are interval numbers that is the probability of occurrence for scenario $k$, with $p_{k} \geq 0$ and $\sum_{k=1}^{K} p_{k}=1 ; \quad \hat{w}_{i k}^{ \pm}$are random variables associated with probability $p_{k}$. In model (1), the decision variables are divided into two subsets. The $x_{j t}^{ \pm}$represents the first-stage variables, which have to be decided before the random variables are disclosed; $y_{j k t}^{ \pm}$are related to the recourse actions against any infeasibilities after uncertainties are disclosed. 
For Model (3), if $x_{j}^{ \pm}$are considered as uncertain inputs, the existing methods for solving inexact linear programming problems cannot be used directly [7]. In this study, an optimized set of target values will be identified by having $\mu_{j}$ in Model (3) be decision variables. Accordingly, let $x_{j}=x_{j}^{-}+\mu_{j} \Delta x_{j}$, where $\Delta x_{j}=x_{j}^{+}-x_{j}^{-}$ and $\mu_{j t} \in[0,1] ; \mu_{j}$ are decision variables that are used for identifying an optimized set of target values $\left(x_{j}^{ \pm}\right)$in order to support the related policy analyses [7]. According to Reference [9], the IP-ITSP model can be transformed into two deterministic submodels that correspond to the lower and upper bounds of the objective function value, respectively.

\section{Application to Ghg-Emission Mitigation in a Regional Electric Power System}

Consider a typical regional-scale electric power system wherein a manager is responsible for allocating energy resources/services from multiple facilities to multiple end-users through multiple technologies within a planning horizon. The decision maker can formulate the problem as minimizing the expected cost of various energy activities in the region over the planning horizon. Moreover, decision makers always seek to control the emissions of environmental pollutants (e.g., sulfur dioxide $\left(\mathrm{SO}_{2}\right)$, nitrogen oxides $\left(\mathrm{NO}_{\mathrm{x}}\right)$, particulate matter $(\mathrm{PM})$ ) and greenhouse gas (GHG) in order to meet the regional environmental requirement [10]. In the energy conversion sector, every conversion technology has an electricity generation target. If the target is not exceeded, a regular cost will be brought to the system; otherwise, the system will be subject to penalties resulted from the extra labor, management, operation and maintenance costs, or capacity expansion and higher costs for imported energy. The future electricity demand during the planning hori- zon is often modeled as an uncertain parameter associated with an interval probability distribution. Most of the other parameters (such as technological efficiency, economic parameters and utilization factors) are expressed as intervals. Therefore, The FP-ITSP approach is considered appropriate for addressing this planning problem. Table 1 presents the available electricity demands under different interval probability distributions. Electricity generation target, environmental pollutants control and the related economic data are shown in Table 2. Besides, coal-fired power has a residual capacity of $1.0 \mathrm{GW}$, natural gas-fired power has a residual capacity of 0.28 GW, hydropower has a residual capacity of $0.26 \mathrm{GW}$. The representative costs and technical data are investigated based on governmental reports and other related literature [4-6,10].

Based on a detailed analysis of the study system, four major sets of objectives were considered when modeling this system to achieve the following aims: (a) the lowest cost of purchasing coal, nature gas, crude oil, diesel and gasoline, (b) the lowest operation cost for coal-fired power, gas-fired power, hydropower, wind power, solar power, and nuclear power, (c) the lowest capacity expansion cost, and (d) the lowest air pollutant mitigation cost. In detail, the objective functions and constraints were formulated as follows

Table 1. The available electricity demands under different interval probability distributions.

\begin{tabular}{cccc}
\hline \multirow{2}{*}{ Activity } & \multicolumn{3}{c}{ Demand levels } \\
\cline { 2 - 4 } & Low & Medium & High \\
\hline $\begin{array}{c}\text { The amount } \\
\text { of available } \\
\text { electricity }\end{array}$ & {$[105.00,125.00]$} & {$[120.00,145.00]$} & {$[140.00,168.00]$} \\
Probability & {$[0.15,0.35]$} & {$[0.45,0.60]$} & {$[0.05,0.15]$} \\
\hline
\end{tabular}

Table 2. Electricity generation target and the related economic data.

\begin{tabular}{|c|c|c|c|c|c|}
\hline \multicolumn{6}{|c|}{ Electricity generation target and the related economic data } \\
\hline$k=1$ & $k=2$ & $k=3$ & $k=4$ & $k=5$ & $k=6$ \\
\hline \multicolumn{6}{|c|}{ Generation target of each power conversion technology (103GWh) } \\
\hline$[15,100]$ & {$[0,55]$} & {$[0,35]$} & {$[0,25]$} & {$[0,15]$} & {$[0,10]$} \\
\hline \multicolumn{6}{|c|}{ Regular costs for power generation by each power conversion technology $(\$ 106 / 103 G W h)$} \\
\hline$[4.00,6.00]$ & {$[5.00,7.00]$} & {$[5.50,7.50]$} & {$[2.50,3.50]$} & {$[2.00,3.00]$} & {$[10.00,14.00]$} \\
\hline \multicolumn{6}{|c|}{ Surplus costs for power generation by each power conversion technology $(\$ 106 / 103 G W h)$} \\
\hline$[2.00,3.00]$ & {$[2.50,4.50]$} & {$[3.50,5.50]$} & {$[1.50,2.50]$} & {$[1.00,2.00]$} & {$[6.00,9.00]$} \\
\hline \multicolumn{6}{|c|}{ Cost of removal SO2 in each power conversion process (\$/tonnes) } \\
\hline$[38.50,45.50]$ & {$[32.50,40.50]$} & 0 & 0 & 0 & 0 \\
\hline \multicolumn{6}{|c|}{ Cost of removal NOx in each power conversion process (\$/tonnes) } \\
\hline$[55.50,75.00]$ & {$[42.50,65.50]$} & 0 & 0 & 0 & 0 \\
\hline \multicolumn{6}{|c|}{ Cost of removal PM2.5 in each power conversion process (\$/tonnes) } \\
\hline$[120.50,135.50]$ & 0 & 0 & 0 & 0 & 0 \\
\hline
\end{tabular}




$$
\begin{aligned}
& \text { Minmizef } \pm(1)+(2)+(3)+(4) \\
&(1)= \sum_{i=1}^{I} Z_{i}^{ \pm} \cdot P E S_{i}^{ \pm} \\
&(2)= \sum_{k=1}^{K} P V_{k}^{ \pm} \cdot W_{k}^{ \pm}+\sum_{k=1}^{K} \sum_{h=1}^{H} p_{h} \cdot\left(P V_{k}^{ \pm}+P P_{k}^{ \pm}\right) \cdot Q_{k h}^{ \pm} \\
& \text {(3) }=\sum_{k=1}^{K} \sum_{m=1}^{M} \sum_{h=1}^{H} p_{h} \cdot Y_{k m h}^{ \pm} \cdot E C_{k m} \cdot I C_{k m} \\
& \text { (4) }=\sum_{k=1}^{K} \sum_{h=1}^{H}\left(W_{k}^{ \pm}+p_{h} \cdot Q_{k h}^{ \pm}\right) \cdot S O T_{k}^{ \pm} \cdot C T S_{k}^{ \pm} \\
&+\sum_{k=1}^{K} \sum_{h=1}^{H}\left(W_{k}^{ \pm}+p_{h} \cdot Q_{k h}^{ \pm}\right) \cdot N O T_{k}^{ \pm} \cdot C T N_{k}^{ \pm} \\
&+\sum_{k=1}^{K} \sum_{h=1}^{H}\left(W_{k}^{ \pm}+p_{h} \cdot Q_{k h}^{ \pm}\right) \cdot P M T_{k}^{ \pm} \cdot C T M_{k}^{ \pm}
\end{aligned}
$$

subject to:

$$
F E_{k}^{ \pm} \cdot\left(W_{k}^{ \pm}+Q_{k h}^{ \pm}\right) \leq Z_{i}^{ \pm}, \forall h
$$

[Mass balance for coal]

$$
\begin{gathered}
\sum_{k=1}^{K}\left(W_{k}^{ \pm}+Q_{k h}^{ \pm}\right)+Z_{1} \geq D T E_{h}^{ \pm}, \forall h \\
W_{k \max }^{ \pm} \geq W_{k} \geq Q_{k h}^{ \pm} \geq 0, \quad \forall k, h \\
W_{k}^{ \pm}+Q_{k h}^{ \pm} \leq S T_{k}^{ \pm} \cdot\left(R C_{k}+\sum_{m=1}^{M} Y_{k m h} \cdot E C_{k m}\right), \quad \forall k, h
\end{gathered}
$$

[Mass balance for electricity generation]

$$
\begin{gathered}
Y_{k m h}^{ \pm}=\left\{\begin{array}{l}
\leq 1 \\
\geq 0 \\
\text { integer }
\end{array} \quad \forall k, m, h\right. \\
\sum_{m=1}^{v} Y_{k m h}^{ \pm} \leq 1, \forall k, h
\end{gathered}
$$

[Capacity expansion]

$$
\begin{gathered}
\sum_{k=1}^{K}\left(W_{k}^{ \pm}+Q_{k h}^{ \pm}\right) \cdot S O T_{k}^{ \pm} \cdot\left(1-\eta_{S}^{ \pm}\right) \leq E S^{ \pm}, \forall h \\
\sum_{k=1}^{K}\left(W_{k}^{ \pm}+Q_{k h}^{ \pm}\right) \cdot N O T_{k}^{ \pm} \cdot\left(1-\eta_{N}^{ \pm}\right) \leq E N^{ \pm}, \forall h \\
\sum_{k=1}^{K}\left(W_{k}^{ \pm}+Q_{k h}^{ \pm}\right) \cdot P M T_{k}^{ \pm} \cdot\left(1-\eta_{O}^{ \pm}\right) \leq E P M^{ \pm}, \forall h \\
\sum_{k=1}^{K}\left(W_{k}+Q_{k h}\right) \cdot C O T_{k}^{ \pm} \leq E M^{ \pm}, \forall h
\end{gathered}
$$

[Environmental constraints]

$$
\beta_{h}^{-} \leq p_{h} \leq \beta_{h}^{+}, h=1,2, \ldots, H
$$

$$
\begin{gathered}
p_{h}=\frac{\sum_{h=1}^{H} \beta_{h}^{+}-1}{\sum_{h=1}^{H} \beta_{h}^{+}-\sum_{h=1}^{H} \beta_{h}^{-}} \cdot \beta_{h}^{-}+\frac{1-\sum_{h=1}^{H} \beta_{h}^{-}}{\sum_{h=1}^{H} \beta_{h}^{+}-\sum_{h=1}^{H} \beta_{h}^{-}} \cdot \beta_{h}^{+} \\
\sum_{h=1}^{H} p_{h}=1
\end{gathered}
$$

where $f^{ \pm}$is the expected system cost (million dollar); $k$ is the electricity conversion technology, $\mathrm{k}=1$ for coal-fired power, 2 for natural gas fired power, 3 for hydropower, 4 for wind-power, 5 for solar power and 6 for nuclear power; $m$ is the capacity expansion option, $m$ $=1,2,3 \ldots, \mathrm{v} ; h$ is the level of power load, $\mathrm{h}=1,2,3 \ldots$, $\mathrm{H} ; Z_{i}^{ \pm}$is the energy sources supply (PJ), $\mathrm{i}=1$ for import electricity, $i=2$ for coal supply, $i=3$ for natural-gas supply, $\mathrm{i}=4$ hydropower supply, $\mathrm{i}=5$ for solar power supply, $\mathrm{i}=6$ for wind power supply, $\mathrm{i}=7$ for nuclear power supply; $P E S_{i}^{ \pm}$is energy sources supply cost (\$million/PJ); $P V_{k}^{ \pm}$is the variable cost for converting technologies $\mathrm{k} ; P P_{k}^{ \pm}$is penalty cost of converting technologies $\mathrm{k}$ for excess electricity generation (\$million/ GWh); $Q_{k h}^{ \pm}$is the excess power generation by technology $\mathrm{k}$ in scenario $\mathrm{h} ; p_{h}$ is the probability of electricity demand; $Y_{k m h}^{ \pm}$is the binary variable for technology $\mathrm{k}$ with expansion option $\mathrm{m} ; W_{k}^{ \pm}$is the allowable power generation by technology $\mathrm{k}$ in load level $\mathrm{h} ; E C_{k m}$ is the capacity expansion size option $\mathrm{m}$ for electricity generation technology $\mathrm{k}(\mathrm{GW}) ; I C_{k m}$ is the capital cost for electricity generation technology $\mathrm{k}$ expansion size $\mathrm{m}$ (\$Million/GW); $\mathrm{COT}_{k}^{ \pm}$is the $\mathrm{CO}_{2}$ emission intensity of power generation technology k (kiloton/GWh); $S O T_{k}^{ \pm}$ is the $\mathrm{SO}_{2}$ emission intensity of power generation technology k (kiloton/GWh); $N O T_{k}^{ \pm}$is the $\mathrm{NO}_{\mathrm{x}}$ emission intensity of power generation technology $k$ (kiloton/ GWh); $P M T_{t}^{ \pm}$is the $\mathrm{PM}_{10}$ emission intensity of power generation technology $\mathrm{k}$ (kiloton/GWh); $C T S_{k}^{ \pm}$is the $\mathrm{SO}_{2}$ removal cost of power generation technology $\mathrm{k}$ (dollar/kiloton); $C T N_{k}^{ \pm}$is the $\mathrm{NO}_{2}$ removal cost of power generation technology $\mathrm{k}$ (dollar/kiloton); $C T M_{t}^{ \pm}$is the $\mathrm{PM}_{10}$ removal cost of power generation technology $\mathrm{k}$ (dollar/kiloton); $F E_{k}^{ \pm}$is the conversion efficiency(TJ/ $\mathrm{GWh}) ; D T E_{h}^{ \pm}$is the electricity demand in load level h (GWh); $R C_{k}$ is the residual capacity of electricity generation technology $\mathrm{k}(\mathrm{GW}) ; \eta_{S}^{ \pm}$is the $\mathrm{SO}_{2}$ removal efficiency of power generation technology $\mathrm{k} ; \eta_{N}^{ \pm}$is the $\mathrm{NO}_{\mathrm{x}}$ removal efficiency of power generation technology $\mathrm{k} ; \eta_{O}^{ \pm}$is the $\mathrm{PM}_{10}$ removal efficiency of power generation technology $\mathrm{k} ; E M^{ \pm}$is the total allowable $\mathrm{CO}_{2}$ emissions (kiloton); $E S^{ \pm}$is the total allowable $\mathrm{SO}_{2}$ emissions (kiloton); $E N^{ \pm}$is the total allowable $\mathrm{NO}_{2}$ emissions (kiloton); $E P M^{ \pm}$is the total allowable $\mathrm{PM}_{10}$ emissions in period t (kiloton). 


\section{Results Analysis}

Table 3 presents the electricity generation targets under different GHG reduction scenarios. As a part of intermediate energy conversion, the electricity generation technologies include coal-fired power, natural gas-fired power, hydropower, wind power, solar power, and nuclear power. Due to the higher cost during the power generation process, the pre-regulated electricity generated by natural gas-fired power, solar power, and nuclear power conversion technologies would always zero under different GHG reduction emission scenarios, and the major power generation technologies include coal-fired power, hydropower, and wind power. The pre-regulated electricity generated by coal- fired power would decrease as the level of GHG-reduction increasing. For example, it would decrease from 42.11× 103 GWh (without GHGreduction) to $16.85 \times 103 \mathrm{GWh}$ (60\% GHG-reduction). Under the four scenarios, the amount of pre-regulated electricity generated by hydropower would be $13.45 \times$ 103GWh, $17.66 \times 103 \mathrm{GWh}, 23.74 \times 103 \mathrm{GWh}$, and $32.16 \times 103 \mathrm{GWh}$ under the scenarios of $0 \%, 20 \%, 40 \%$ and 60\% GHG-reduction emission levels, respectively. The pre-regulated electricity generated by wind power would always $25.00 \times 103 \mathrm{GWh}$ under different GHG reduction emission scenarios.
If the electricity targets cannot meet the random demand, during different excess GHG reduction emission scenarios, electricity has to be produced under different demand levels. The excess generation quantities of each power conversion technology would be different from under different demand levels and different GHG-reduction levels as shown in Table 4. In case of insufficient electricity supply, wind power would be firstly as the recourse activities to compensate the deficits of electricity shortages, the coal-fired power and hydropower would only be supplements. Moreover, as the electricity demand level increasing, excess electricity generated by the three power technologies would increase. For example, under 0\% GHG-reduction level, the excess electricity generated by wind power technologies would be 0 , [0, 1.95] $\times 103 \mathrm{GWh}$, and [0, 11.5] × $103 \mathrm{GWh}$ during the low, medium, and high level of electricity demand, respectively. In addition, as the GHG-reduction level increasing, the excess power generation would also increase, for example, under the high level of electricity demand, the excess electricity generated by hydropower technologies would be $13.45 \times 103$ GWh, $17.66 \times 103$ GWh, $20.00 \times 103 \mathrm{GWh}$, and [20.00, 21.81] × $103 \mathrm{GWh}$ during $0 \%, 20 \%, 40 \%$ and $60 \%$ of GHG-reduction emission levels, respectively.

Table 3. Solutions of electricity generation targets under GHG reduction scenarios.

\begin{tabular}{|c|c|c|c|c|c|c|}
\hline \multirow{2}{*}{$\begin{array}{l}\text { GHG-emission } \\
\text { reduction level }\end{array}$} & \multicolumn{6}{|c|}{ Electricity generation targets (103 GWh) } \\
\hline & $\begin{array}{l}\text { Coal-fired power } \\
\qquad(k=1)\end{array}$ & $\begin{array}{l}\text { Natural gas-fired power } \\
\qquad(k=2)\end{array}$ & $\begin{array}{l}\text { Hydropower } \\
\quad(k=3)\end{array}$ & $\begin{array}{l}\text { Wind-power } \\
\quad(k=4)\end{array}$ & $\begin{array}{l}\text { Solar power } \\
\quad(k=5)\end{array}$ & $\begin{array}{l}\text { Nuclear power } \\
\quad(k=6)\end{array}$ \\
\hline $0 \%$ & 42.11 & 0 & 13.45 & 25.00 & 0 & 0 \\
\hline $20 \%$ & 33.68 & 0 & 17.66 & 25.00 & 0 & 0 \\
\hline $40 \%$ & 25.26 & 0 & 23.74 & 25.00 & 0 & 0 \\
\hline $60 \%$ & 16.85 & 0 & 32.16 & 25.00 & 0 & 0 \\
\hline
\end{tabular}

Table 4. Solutions of the excess power generation of each conversation technology.

\begin{tabular}{|c|c|c|c|c|c|c|c|}
\hline \multirow{2}{*}{ Level } & \multirow{2}{*}{$\mathrm{CO}_{2}$ emission reduction level } & \multicolumn{6}{|c|}{ Excess power generation of each conversation technology $\left(10^{3}\right.$ GWh) } \\
\hline & & $k=1$ & $k=2$ & $k=3$ & $k=4$ & $k=5$ & $k=6$ \\
\hline \multirow{4}{*}{$\mathrm{L}$} & $0 \%$ & 0 & 0 & 0 & {$[3.45,6.95]$} & 0 & 0 \\
\hline & $20 \%$ & 0 & 0 & 0 & {$[7.66,11.16]$} & 0 & 0 \\
\hline & $40 \%$ & 0 & 0 & 0 & {$[10.00,13.50]$} & 0 & 0 \\
\hline & $60 \%$ & 0 & 0 & 0 & {$[10.00,13.50]$} & 0 & 0 \\
\hline \multirow{4}{*}{ M } & $0 \%$ & {$[0,1.95]$} & 0 & 0 & {$[18.45,25.00]$} & 0 & 0 \\
\hline & $20 \%$ & {$[0,6.16]$} & 0 & 0 & {$[22.66,25.00]$} & 0 & 0 \\
\hline & $40 \%$ & {$[0,8.50]$} & 0 & 0 & 25.00 & 0 & 0 \\
\hline & $60 \%$ & {$[0,8.50]$} & 0 & 0 & 25.00 & 0 & 0 \\
\hline \multirow{4}{*}{$\mathrm{H}$} & $0 \%$ & {$[0,11.50]$} & 0 & 13.45 & 25.00 & 0 & 0 \\
\hline & $20 \%$ & {$[0,11.50]$} & 0 & 17.66 & 25.00 & 0 & 0 \\
\hline & $40 \%$ & {$[0,11.50]$} & 0 & 20.00 & 25.00 & 0 & 0 \\
\hline & $60 \%$ & {$[0,9.69]$} & 0 & {$[20.00,21.81]$} & 25.00 & 0 & 0 \\
\hline
\end{tabular}


As shown in Figure 1, compared the six power generation technologies' contribution to the different levels of electricity demand, it indicates that different power conversion technologies have varied generation quantities under changed GHG-emission reduction scenarios. As the previous section analysis, natural gas-fired power,
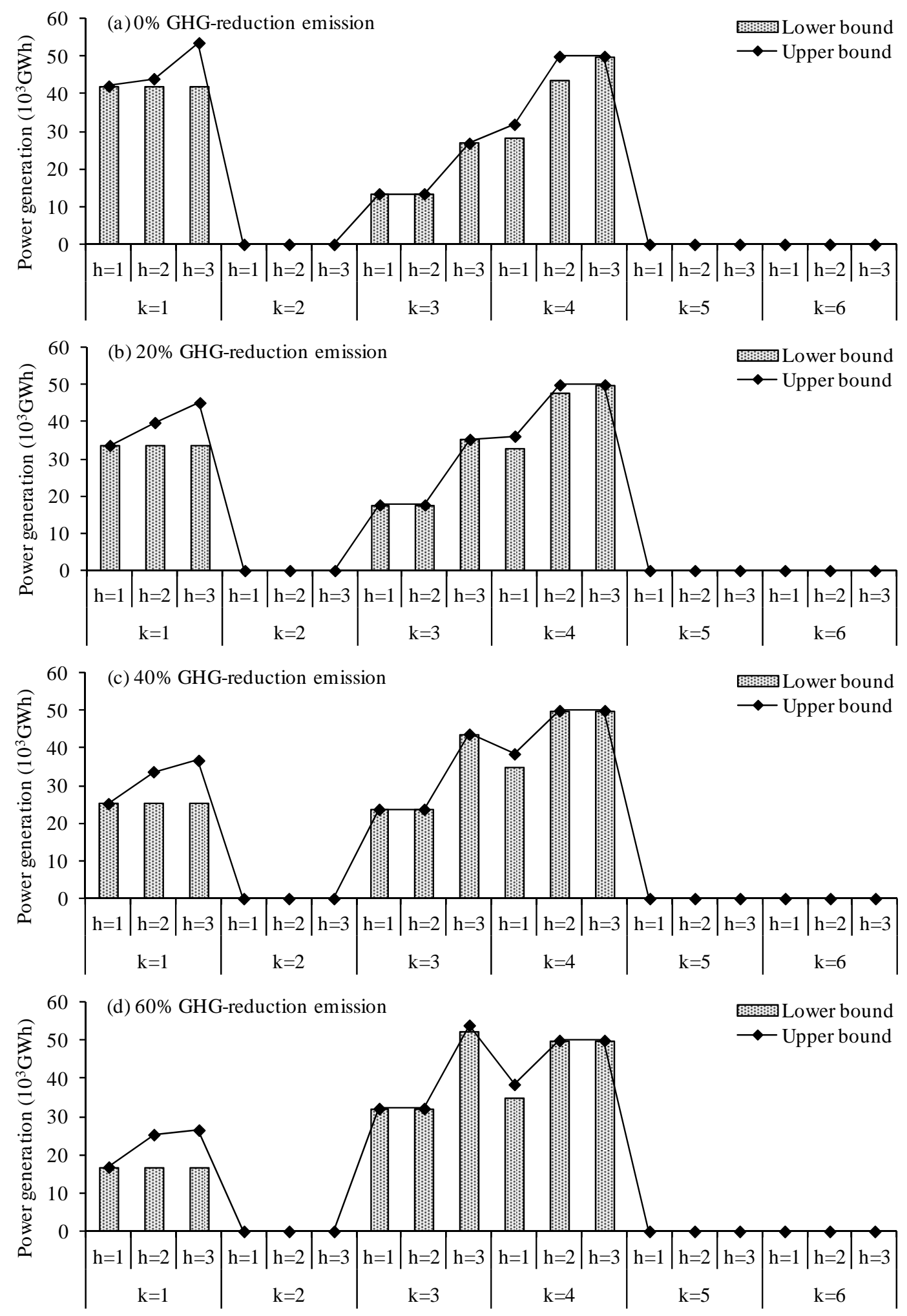

Figure 1. Amount of power generation with $0 \%, 20 \%, 40 \%$ and $60 \%$ GHG reduction. 
solar power and nuclear power would be zero under different levels of GHG-reduction emission. This is because nuclear power would enhance the diversity of power generation, and thus increase the stability and security of the study system, solar power would be limited by space and resource during the study area, and the related cost of gas-fired power conversion technology is slightly higher than coal-fired power. Under 0\% of GHG-reduction emission, coal-fired power would be the most important electricity supply source, wind power would be the most important electricity generation technologies under $20 \%$, $40 \%$, and $60 \%$ GHG-emission reduction. As the GHGemission reduction level increasing, the total amount of coal-fired power would decrease, especially, under the scenario of $60 \%$ GHG-emission reduction, the hydropower generation would be bigger than coal-fired power. For example, under $60 \%$ GHG-emission reduction, the total power generation of coal-fired power technologies would be $16.84 \times 103 \mathrm{GWh},[16.84,25.34] \times 103 \mathrm{GWh}$, and $[16.84,26.53] \times 103 \mathrm{GWh}$ in the scenarios of low, medium, and high demand level, respectively; the hydropower would be $32.16 \times 103 \mathrm{GWh}, 32.16 \times 103 \mathrm{GWh}$, and $[52.16,53.97] \times 103 \mathrm{GWh}$ under the three demand levels; the amount of wind power generation would [35.00, 38.50] × $103 \mathrm{GWh}, 50.00 \times 103 \mathrm{GWh}, 50.00 \times$ 103 GWh under the low, medium, and high level of electricity demand, respectively. It indicates that although coal-fired power conversion technology has relatively low operating and penalty costs and comparatively low capital cost for capacity expansion, it has a higher GHGemission during the electricity generation process; more and more environment-friendly power conversion technologies would be chosen for electricity generation to satisfy the ever-increasing electricity demands and enhancing GHG-emission reduction requirements.

Moreover, imported power would be purchased to fill the power shortage by the energy system decision makers. In this study, in order to enhance the regional power system reliability, it assumes that $[20,30] \%$ of the total electricity demand would be the maximum amount of imported power. Under different scenarios, because of the due to lower cost, the imported electricity would reach to the maximum limitation, being [21.00, 37.50] × 103GWh. As shown in Figure 2, the system cost would rise up along with increasing GHG-emission reduction. The system cost would be $\$[491.84,727.52] \times 106, \$$ [500.57, 744.41] × 106, \$ [510.91, 759.24] × 106, and \$ [523.26, 772.37] $\times 106$ under $0 \%, 20 \%, 40 \%$, and $60 \%$ GHG-reduction, respectively. The cost of GHG mitigation (per kiloton) would increase, being $\$[1.09,1.30] \times$ $106, \$[1.19,1.38] \times 106$, and $\$[1.15,1.31] \times 106$, under $20 \%, 40 \%$, and $60 \%$ GHG-emission reduction, respectively. It indicates that under the scenarios of GHG-reduction, the coal-fired power generation technologies with lower cost in electricity generation process would be replaced by hydropower, wind power which has a higher operation cost, besides, the increasing electricity demand leads to various power generating facilities to be

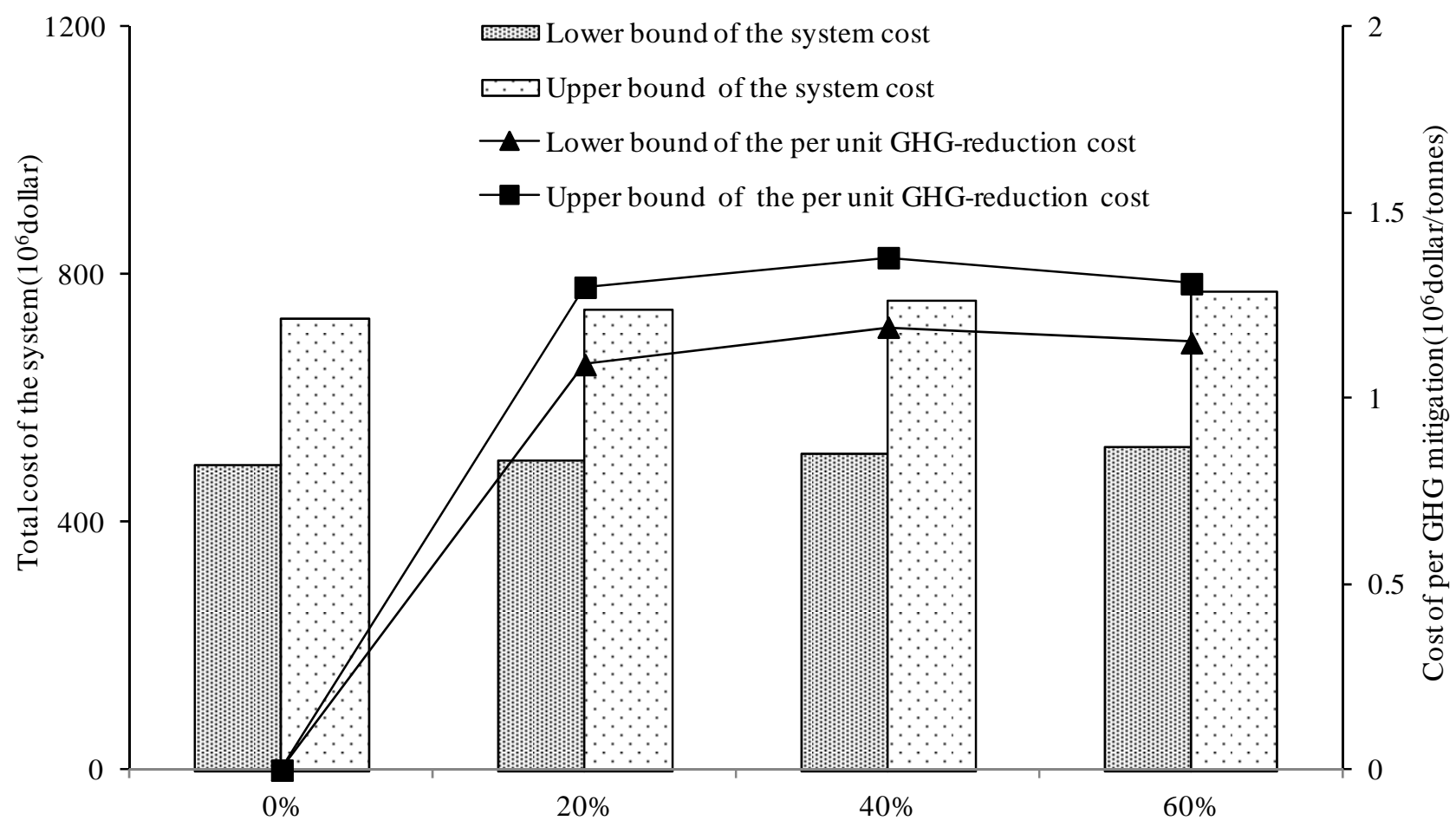

Figure 2. Costs under different GHG reduction scenarios. 
expanded, bringing about a high capital cost.

\section{Conclusion}

An interval probability-based inexact two-stage stochastic (IP-ITSP) model is developed for regional electricity system and greenhouse gas (GHG) emissions reduction management under uncertainties. The method is based on interval probability, interval-parameter programming (IPP) and two-stage stochastic programming (TSP). Uncertainties in the energy system could present as both interval probability distributions and interval values to be incorporated within a general optimization framework. The developed model is applied to a case of planning GHG-emission mitigation in a regional electricity system, demonstrating that IP-ITSP is applicable to reflecting complexities of multi-uncertainty, and capable of addressing the problem of GHG-emission reduction. 4 scenarios corresponding to different GHG -emission mitigation levels are examined; Solutions provide an effective linkage between the predefined environmental policies and the associated economic implications (e.g., losses and penalties caused by improper policies). The solutions contain a combination of deterministic, interval and distributional information, and can thus facilitate the reflection for different forms of uncertainties. The results indicate that the model can help managers obtain multiple decision alternatives, as well as provide bases for further analyses of tradeoffs between energy management cost and GHG-emission reduction.

\section{REFERENCES}

[1] C. B. Field and M. R. Raupach, "The Global Carbon Cycle: Integrating Humans, Climate, and the Natural World,” Island Press, 2004.

[2] IPCC, "Climate Change Contribution of Working Group I to the Fourth Assessment Report of the IntergovernMental Panel on Climate Change,” Cambridge, United King-
dom/New York, NY, USA: Cambridge University Press, 2007.

[3] S. E. Fleten and T. K. Kristoffersen, "Short-term HydroPower Production Planning by Stochastic Programming," Computers and Operations Research, Vol. 35, No. 8, 2008, pp. 2656-2671. doi:10.1016/j.cor.2006.12.022

[4] Q. G. Lin and G. H. Huang, "IPEM: An interval-parameter energy systems planning model,” Energy Sources A: Recovery, Utilization, and Environmental Effects, Vol.30, No.14, 2008, pp.1382-1399. doi:10.1080/15567030801929043

[5] Y.Y. Liu, “A dynamic two-stage energy systems planning model for Saskatchewan, Canada,” M. S. Dissertation, Faculty of Graduate Studies and Research, Regina, Saskatchewan, Canada. 2007.

[6] L. Liu, G. H. Huang, G. A. Fuller, A. Chakma and H. C. Guo, “A Dynamic Optimization Approach for Nonrenewable Energy Resources Management Under Uncertainty," Journal of Petroleum Science and Engineering, Vol. 26, 2000, pp. 301-309. doi:10.1016/S0920-4105(00)00044-9

[7] Y. P. Li, G. H. Huang, S. L. Nie, X. H. Nie and I. Maqsood, "An Interval-parameter Two-stage Stochastic Integer Programming Model for Environmental Systems Planning under Uncertainty,” Engineering Optimization, Vol. 38, No. 4, 2006, pp. 461-483. doi:10.1080/03052150600557742

[8] R. R. Yager, "Decision Making under Interval Probabilities,” International Journal of Appeoximate Reasoning, Vol. 22, No. 3, 1999, pp. 195-215. doi:10.1016/S0888-613X(99)00028-6

[9] G. H. Huang and D. P. Loucks, “An Inexact Two-stage Stochastic Programming Model for Water Resources Management under Uncertainty," Civil Engineering and Environmental Systems, Vol. 17, 2000, pp. 95-118. doi:10.1080/02630250008970277

[10] Y. L. Xie, Y. P. Li, G. H. Huang and Y. F. Li, “An Interval Fixed-mix Stochastic Programming Method for Greenhouse Gas Mitigation in Energy Systems under Uncertainty,” Energy, Vol. 35, 2010, pp. 4627-4644. doi:10.1016/i.energy.2010.09.045 